\title{
Biomedical Sleep Inducer System
}

\author{
Deepjyoti Mahanta \\ Assam Don Bosco University \\ Guwahati \\ deepjyoti94@yahoo.com
}

\author{
Hemashree Bordoloi \\ Assam Don Bosco University \\ Guwahati \\ hemashree.bordoloi@dbuniversity.ac.in
}

\author{
Samar Jyoti Saikia \\ Assam Don Bosco University \\ Guwahati \\ samar_saikia@yahoo.com
}

\begin{abstract}
Sleeping difficulty called insomnia, can involve difficulty in falling asleep one who has first go to bed at night, waking up too early in the morning and waking up often during night. The lack of restful sleep can affect your ability to hold out daily responsibilities. All types of insomnia can cause day time drowsiness, poor concentration, and therefore the inability to feel refreshed and rested in the morning. Magnetic flux related to the plannet is termed geo-magnetic fields. It is essentially dipolar on the earth's surface. Many of us experience sleeping well within the natural surroundings into a tent or a wooden hut. This fact is because of not only to the healthy atmosphere but also from our unconscious ability to perceive natural earth's magnetic fields. Our paper is about this sort of geo-magnetic -fields. This has been designed a circuit, which radiates an electromagnetic field which is low frequency through a radiator coil and our aim is to perceive them, in this manner our brain is surrounded by a perfect environment for a sound sleep.
\end{abstract}

Keywords - Sleep inducer, insomnia, geo magnetic field.

\section{INTRODUCTION}

Stress, a common occurrence and affecting almost all the ages of human body due to sev- eral external and internal factors like work load, relationship difficulties, chronic worry etc. has evolved as a threat to various physiological or biological activities thus influenc- ing human behavior and leading to Insomnia. According to World Health Organization, stress has become a 'World Wide Epidemic'[1].

Magnets have been used for centuries to treat a number of physical disorders. We have designed a circuit which creates and radiates an electromagnetic field through a radiator coil and creates an environment helpful for sound sleep. A theory of accelerated transition from wakefulness to sleep is proposed to explain the process of insomnia relief through low-strength static magnetic fields [2].Other discovered brainwave entertainment decades ago using binaural beats (sounds) and pulsating light, although it's hard to sleep with these systems due to obvious limitations. Our objective is to design a tool which can create an equivalent pattern of wave that makes in brain during sleep. It generates an electromagnetic-field, makes easier to fall asleep, and induces a prolonged and sound sleep without drugs [3] [4].

\section{LITERATURE SURVEY}

\section{A. Causes of insomnia}

- Life events like fear, stress, anxiety, emotional or mental tension, work problems, financial stress.

\section{B. Symptoms of insomnia}

\section{Night-time Symptoms:}

- Frequent difficulty falling asleep.
- Frequent episodes of walking up accompanied by difficulty in falling back to sleep.

\section{Insomnia \& Its different stages}

Insomnia or sleeplessness is a human reported sleeping difficulties. A definition of insomnia is, Difficulties initiating and maintaining sleep, or non- restorative sleep, related to impairments of daytime functioning or marked distress for more over 1 month.

Insomnia can occur at any age, but it is particularly common within the elderly. Insomnia are often transient insomnia (lasts for less than a week) or Acute insomnia (means inability to consistently sleep well for a period of less than a month) or chronic insomnia (lasts for extended than a month) which may cause memory problems, depression, irritability and an increased risk of heart condition and automobile related accidents etc.

D. Categories of Brain Wave Pattern

$>\operatorname{Beta}(14-30 \mathrm{~Hz})$

- Concentration, arousal, alertness, cognition

- Higher levels associated with anxiety, disease, feelings of sparation, fight [5].

$>\operatorname{Alpha}(8-13.9 H z$

- Relaxation, super learning, relaxed focus, increased serotonin production [5]

$>$ Theta $(4-7.9 H z)$

- Dreaming sleep (REM sleep)

- Increased production of amines, increased creativity [5]

\section{$>\operatorname{Delta}(1-3.9 H z)$}

- Dreamless sleep [5] [11]

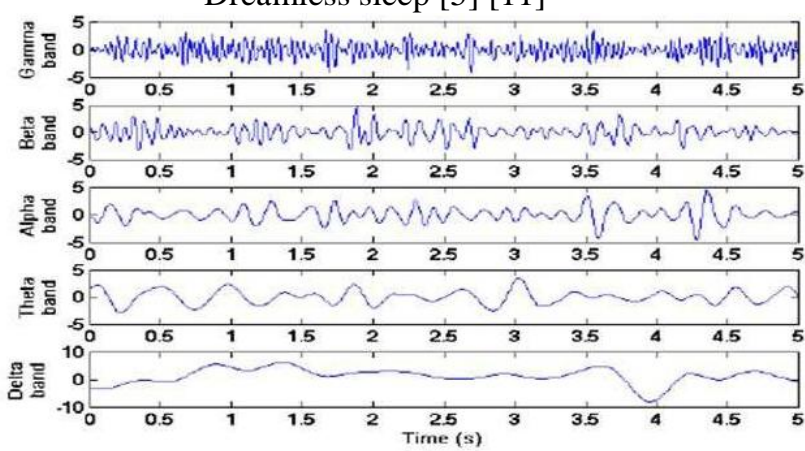

Fig. 1. Classification of Brain Signals

\section{E. Condition of Human Brain during Sleep}

When we close our eyes and relax, the predominant EEG pattern will be a slow oscillation between about 7 and 12 
hertz. This waveform is termed the alpha wave, and is related to contentment and a decreased level of attention. Opening our eyes and looking around causes the EEG to change to the beta rhythm, occurring between about $17 \mathrm{~Hz}$ and $20 \mathrm{~Hz}$. Other frequencies and waveforms are seen in children, different depths of sleep, and various brain disorders such as epilepsy, insomnia etc [7].

When we first enter sleep, our brain waves decelerate from beta (12 to 18 cycles/second) to alpha (8 to 12 cycles/second) to theta (4 to 8 cycles/second). At this point, stage one begins. The amplitude of sleep waves is lowest during wakefulness $(10-30 \mu \mathrm{V})$ and shows a progressive increase through the various stages of sleep [5] [6].

\section{METHODOLOGY}

Faradays law of electromagnetic induction is that the main principle of this device. Which could be a basic law of electromagnetism predicting how a magnetic flux will interact with an electrical circuit to produce an electromotive force (EMF) a phenomenon called electromagnetic induction [8].

The brain is usually generating a pattern of internal neural frequencies, so called alpha, theta, delta, and beta; names for various ranges of frequencies, plus others, some of which are altered by the patterns of electromagnetism in our environment. Radio waves, cellular phone microwaves, TV, and general noise from electric circuits also generate electromagnetic frequencies. The minute electromagnetic patterns of the Earth are also a part of the environment [9] [10]

The world is surrounded by magnetic fields: some generated by the earth's magnetism, others generated by solar storms and changes in weather. Magnetic fields are created by electrical devices (e.g. motors, televisions, office equipment, computers, microwave ovens, electrical wiring in homes, power lines). Even the human body produces a subtle magnetic fields generated by chemical change within cells and ionic currents of the nervous system. An electromagnetic field (EMF) is consists of both an electrical and a magnetic flux. The electrical field is because of the presence of charged particles (such as electrons) and therefore the magnetic flux is because of the movement of the charged particles (such as an electron current). Recently, scientists discover that external magnetic fields affect the body's functioning in both different ways [8].

\section{DESIGN OF THE BIOMEDICAL SLEEP INDUCER SYSTEM CIRCUIT}

The main function of the Biomedical Sleep Inducer System is to produce a ELF (Extra low frequency, here9.6 $\mathrm{Hz}$ ) signal which in turn drive a electromagnet to produce a electromagnetic field of the same frequency.Fig .2 shows the circuit diagram of the sleep inducer system using IC555 and BC547.

Pin 1 of the Timer IC is connected to ground while Trigger (pin2) and Threshold (Pin6) are common with a 0.5 ${ }^{I} \mathrm{~F}$ capacitor which are directly connected to Ground from that common point. From discharge (pin 7) a resistor of 100 $\mathrm{K}$ - is connected to the Vcc. A resistor of $100 \mathrm{~K}$ - is connected between the Pin 7 and Pin 6 . This will drop the voltage $1 / 3$ of the Vcc and hence trigger will be operated. While Vcc (pin8) is common with RESET (pin4) and hence Circuit starts works with resetting the previous value.

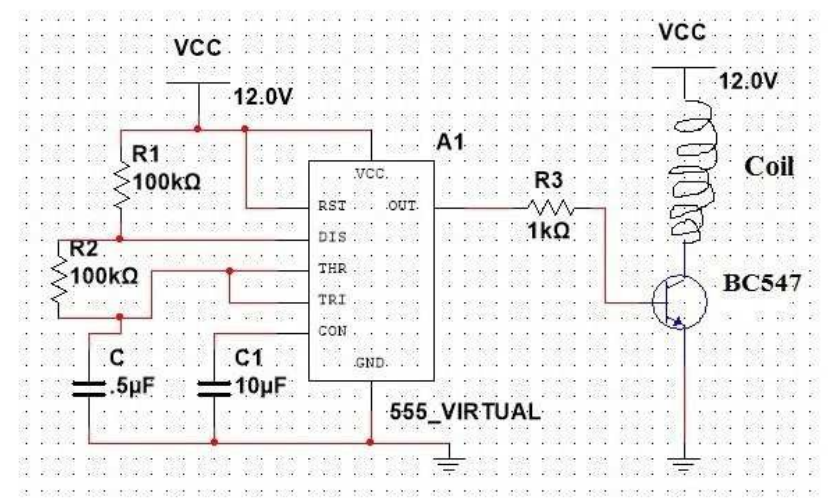

Fig. 2. Circuit Diagram of the Sleep Inducer System using IC555 and BC547

Theoretical on time is calculated using equation (1) and the off time is calculated using equation (2) [12]

$$
\begin{gathered}
T_{\text {ON }}=0.69\left(R_{1}+R_{2}\right) C \\
T_{\text {OFF }}=0.69\left(R_{2}\right) C \\
T_{\text {TOTALTIME }}=0.69\left(R_{1}+2 R_{2}\right) C \\
\% \text { dutycycle }=T_{\text {ON }} / T_{\text {TOTALTIME }} \times 100 \\
\text { Frequency }=1.44 /\left(R_{1}+2 R_{2}\right) C
\end{gathered}
$$

Here, $\mathrm{R} 1=100 \mathrm{k} \Omega, \mathrm{R} 2=100 \mathrm{k} \Omega, \mathrm{C}=0.5 \mu \mathrm{F}$

Therefore,

$$
\begin{aligned}
& T_{\text {ON }}=69 \mathrm{sec} T_{\text {OFF }}=34.5 \mathrm{sec} \\
& T_{\text {TOTALTIME }}=103.5 \mathrm{sec} \\
& \text { Dutycyclein } \%=66 . .67 \% \\
& \text { Frequency }=9.6 \mathrm{~Hz}
\end{aligned}
$$

From the 555 timer we have generated $9.6 \mathrm{~Hz}$ frequency. We have driven this $9.6 \mathrm{~Hz}$ frequency through a coil using transistor BC547. BC547 is an NPN Bipolar Junction Transistor(BJT). A transistor, stands for transfer of resistance, is usually used to amplify current. A tiny low current at its base controls a larger current at collector and emitter terminals. BC547 is especially used for amplification and switching purposes. It is a maximum current gain of 800 . The transistor terminals require a set of DC voltage to control within the desired region of its characteristic curves. This is called biasing. For amplification applications, the transistor is biased such it is partly on for all input conditions. The input at base is amplified and brought at the emitter. BC547 is employed in Common Emitter(CE) configuration for amplifiers. The potential divider is that the commonly used biasing mode. For switching applications, transistor is biased in order that it remains fully on if there is an indication at its base. Within the absence of base signal, it gets completely off. After we drive this $9.6 \mathrm{~Hz}$ frequency through a coil using transistor $\mathrm{BC} 547$, it generates the $9.6 \mathrm{~Hz}$ electromagnetic frequency. When this transistor is fully biased then it can allow a maximum of $100 \mathrm{~mA}$ to flow across the collector and emitter. This stage is termed Saturation Region and thus the standard voltage allowed across the Collector- Emitter (VCE) or Base-Emitter (VBE) might be $200 \mathrm{mV}$ and $900 \mathrm{mV}$ 
respectively. When base current is removed the transistor becomes fully off, this stage is termed as the Cut-off Region and therefore the Base Emitter voltage might be around $660 \mathrm{mV}$. Using BC547 in this sleep inducer circuit we got a retardant that the transistor heat up because the current that flows through the collector pin is over than $100 \mathrm{~mA}$ but less than $150 \mathrm{~mA}$. If we replace transistor BC547 to transistor BD115 then this problem will solved because when the transistor BD115, is fully biased then it can allow a maximum of $150 \mathrm{~mA}$ to flow across the collector and emitter. Fig 3 shows the Circuit diagram of the sleep inducer circuit using IC555 and transistor BD115.

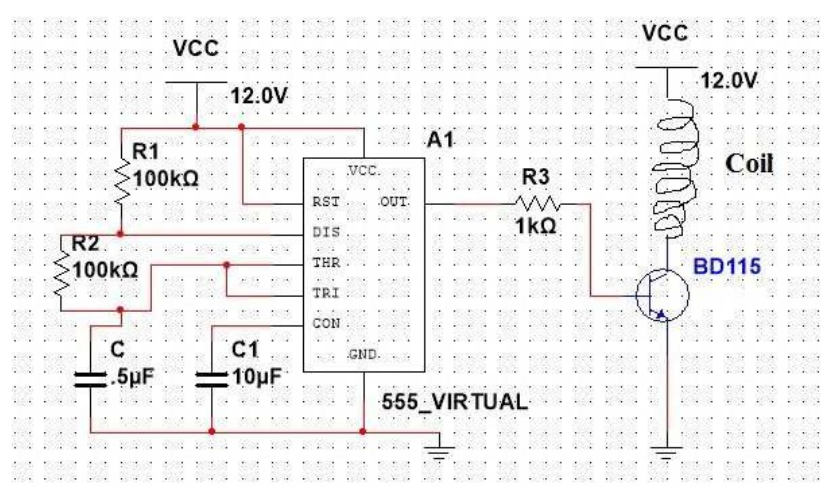

Fig. 3. Circuit Diagram of the Sleep Inducer System using IC555 and BD115

Fig. 4 shows the Hardware implementation of the Sleep Inducer System

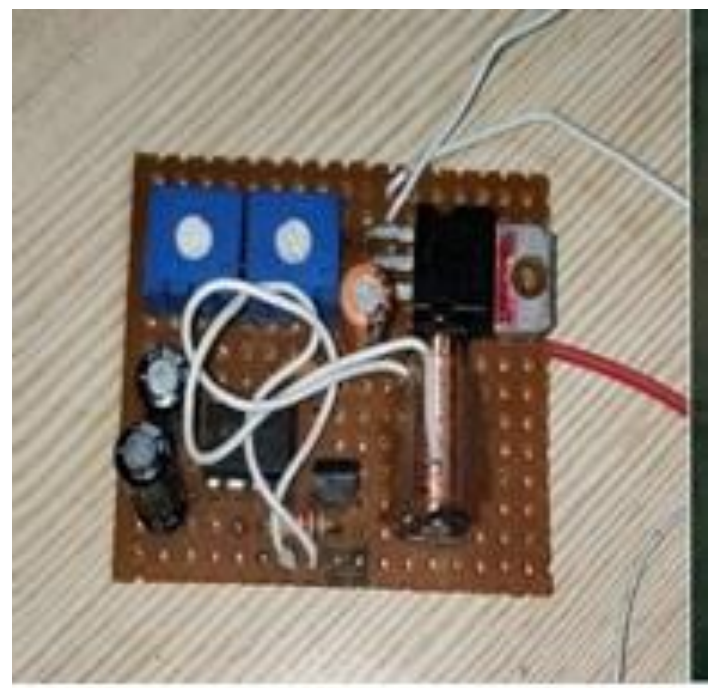

Fig. 4. Hardware implementation of the Sleep Inducer

\section{TESTING OF THE BIOMEDICAL SLEEP INDUCER SYSTEM}

Biomedical Sleep inducer system designed has been tested on the subjects who were facing sleeping problems. Insomnia can be defined as the disorder where the individual is unable to attain sleep, or maintain a sound sleep as well waking up early without a peaceful sleep can also be termed as Insomnia. So testing has been done on the 30 subjects of different age group normally on students. Out of 30 subjects 18 have attained sleep while 5 have no idea whether it worked or not and the rest of 7 subjects didn't gain any differnce in their sleeping habits.

\section{ADVANTAGE}

- This Biomedical Sleep Inducer System helps in fighting insomnia

- This Biomedical Sleep Inducer System generates kind of geo-magnetic fields and it helps the brain surrounded by a perfect environment for a sound sleep.

\section{CONCLUSION AND FUTURE WORK}

This Biomedical Sleep Inducer System helps in fighting insomnia. Apart from this it also supports relaxation, stress management and induces sleep easily without medication. The designed System generates type of geo-magnetic fields and it helps the brain surrounded by an ideal environment for a sound sleep. This system can be improved more.

\section{REFERENCES}

[1] https://thriveglobal.com/stories/stress-the-health-epidemic-of-the21st-century/

[2] S.K.Rits Combining Neural Networks for Protein Secondary Structure Prediction. IEEE 0-7803-2768-3/95/4.00 0.

[3] Sumukh S R, Dimpal kumara, Aishwarya M N, Priya S B, "Biomedical Sleep Inducer", Int. J. Advanced Networking and Applications, Volume No: 8, Issue No: 4(Jan-Feb 2017), Special Issue-NCBSI-2016.

[4] Md.Mahadi Hasan, "Design a Sleep Inducer System”, International Journal of Engineering Research and Technology (IJERT), page 2412, ISSN: 2278-0181, Vol. 3 Issue 5, May - 2014.

[5] A.Saidatult Et al," Analysis of EEG signals during relaxation and mental stress condition using AR modeling techniques", IEEE International Conference on Control System, Computing and Engineering, pp. 477-481,2011.

[6] Z. Zhang et al., "Reduction in time-to-sleep through EEG based brain state detection and audio stimulation," 2015 37th Annual International Conference of the IEEE Engineering in Medicine and Biology Society (EMBC), Milan, 2015, pp. 8050- 8053. doi: 10.1109/EMBC.2015.7320261

[7] Z. Zhang et al., "Automatic sleep onset detection using single EEG sensor," 2014 36th Annual International Conference of the IEEE Engineering in Medicine and Biology Society, Chicago, IL, 2014, pp. 2265-2268.

[8] Y. Morita, K. Yamaguchi, K. Ashida, R. Ikeura andK. Yokoyama, "Verification of sleep-inducing effect by excitation apparatus simulating mother's embrace and rocking motion," Robot Motion and Control (RoMoCo), 2013 9th Workshop on, Kuslin, 2013, pp. 80-85. doi: 10.1109/RoMoCo.2013.6614588

[9] F. Jie, W. Tiecheng, Y. Yan, B. H. Duc and L. Xiaoping, "A magnetic field projector for deep brain modulation," Neural Engineering (NER), 2013 6th International IEEE/EMBS Conference on, San Diego, CA, 2013, pp. 1214-1217.

[10] Marshall L, Helgadóttir H, Mölle M, Born J., Boosting slow oscillations during sleep potentiates memory, (University of Lübeck, Department of Neuroendocrinology

[11] Andrew A. Marino, Erik Nilsen, Andrew L. Chesson Jr., Clifton Frilot, .Consistent magnetic-field induced dynamical changes in rabbit brain activity detected by recurrence quantification analysis

[12] R. A. Gayakwad Op-Amps and Linear integrated Circuits, PHI Private Limited, Fourth Edition. 